\title{
Role of Serum Hepatitis B Virus Marker Quantitation to Differentiate Natural History
}

\section{Lili Wang*}

Infectious Disease Hospital of Yantai, 62 Huanshan Road, Zhifu, Yantai, Shandong, China

"Corresponding author: Dr. Wang L, Infectious Disease Hospital of Yantai, 62 Huanshan Road, Zhifu District, Yantai, 264001, Shandong, China, Tel: +86 0535623225; Email: liliwang2200@163.com

Rec Date: Oct 19, 2015; Acc Date: Dec 10, 2015; Pub Date: Dec 22, 2015

Copyright: @ 2015, Wang L. This is an open-access article distributed under the terms of the Creative Commons Attribution License, which permits unrestricted use, distribution, and reproduction in any medium, provided the original author and source are credited.

\section{Commentary}

In the past decade, a growing body of evidence has shown that HBsAg quantification (qHBsAg) not only is a useful marker for monitoring natural history of treatment-naïve Chronic HBV Infection (CHB) but can also predict clinical and treatment outcomes [1]. While, other study indicated that, being used alone, qHBsAg is not a suitable marker for evaluating hepatitis activity and distinguishing between cases of $\mathrm{HBeAg-negative} \mathrm{CHB}$ and inactive HBV carrier state [2]. In our study, we showed that $\mathrm{qHBsAg}$ had high predictive value for discrimination of immune tolerance (IT) and Immune Clearance (IC) phase, and it had moderate predictive value for differentiation of low replication (LR) phase and HBeAg Negative Hepatitis (ENH) [3]. While, role of $\mathrm{HBeAg}$ quantification $(\mathrm{qHBeAg})$ in natural phases of $\mathrm{HBV}$ infection has not been attracted more attention up to now. We found that in $\mathrm{HBeAg}$ positive phase of treatment-naïve $\mathrm{CHB}$, qHBsAg and $\mathrm{qHBeAg}$ correlated positively $(\mathrm{P}<0.0001)$, and both had strong negative correlation with grade of liver inflammation $(G)(P<0.0001)$ and Fibrosis stage $(\mathrm{F})(\mathrm{P}<0.0001)$ (unpublished data). Thus, we might speculate that $\mathrm{qHBeAg}$ can either be used as a marker of natural phases of $\mathrm{HBV}$ infection in $\mathrm{HBeAg}$ positive patients. Indeed, we demonstrated that $\mathrm{qHBeAg}$ had moderate predictive value for discriminating IT and IC phase [3]. And qHBsAg and qHBeAg had moderate predictive value for F1, F2 and F3 and for G2 and G3 in treatment-naïve $\mathrm{HBeAg}$ positive $\mathrm{CHB}$ (unpublished data). In recent years, $\mathrm{HBcAb}$ quantification $(\mathrm{qHBcAb})$ has been indicated to be associated with HBV infection induced hepatitis [4]. We also suggested that $\mathrm{qHBcAb}$ are higher in IC and ENH phases than in IT and LR phases. And $\mathrm{qHBcAb}$ correlated positively with grade of liver inflammation. Whereas, the $\mathrm{qHBcAb}$ has low divisional value for the intermediate grades of inflammation (unpublished data). Next, we will determine whether $\mathrm{qHBcAb}$ is different with liver injury of different etiologies in HBV infection, including HBV infection merged with non-alcoholic fatty liver disease or combined with drug induced liver injury. In all, qHBsAg had high and $\mathrm{qHBeAg}$ had moderate predictive value for discrimination of IT and IC phase. And both had moderate predictive value for diagnosis of mild to severe liver fibrosis in treatment-naïve $\mathrm{HBeAg}$ positive $\mathrm{CHB}$. Both qHBsAg and qHBcAb had moderate predictive value for differentiation of LR and ENH phase.

\section{References}

1. Martinot-Peignoux M, Lapalus M, Asselah T, Marcellin P (2014) HBsAg quantification: useful for monitoring natural history and treatment outcome. Liver Int 1: 97-107.

2. Tan Z, Li M, Kuang X, Tang Y, Fan Y, et al. (2014) Clinical implications of hepatitis B surface antigen quantitation in the natural history of chronic hepatitis B virus infection. J Clin Virol 59: 228-234.

3. Wang L, Zou ZQ, Wang K, Yu JG, Liu XZ (2015) Role of serum hepatitis $B$ virus marker quantitation to differentiate natural history phases of HBV infection. Hepatol Int

4. Yuan Q, Song LW, Cavallone D, Moriconi F, Cherubini B, et al. (2015) Total Hepatitis B Core Antigen Antibody, a Quantitative Non-Invasive Marker of Hepatitis B Virus Induced Liver Disease. PLoS One 10: e0130209. 